\title{
Ignoring the Elephant in the Room: A Neural Circuit to Downregulate Salience
}

\author{
Carmel Mevorach, ${ }^{1}$ John Hodsoll, ${ }^{1}$ Harriet Allen, ${ }^{1}$ Lilach Shalev, ${ }^{2}$ and Glyn Humphreys ${ }^{1}$ \\ ${ }^{1}$ Behavioural Brain Sciences Centre, School of Psychology, University of Birmingham, Birmingham B15 2TT, United Kingdom, and ${ }^{2}$ Division of Learning \\ Disability, School of Education, Hebrew University of Jerusalem, Jerusalem 91905, Israel
}

\begin{abstract}
How do we ignore stimuli that are salient but irrelevant when our task is to select a lower salient stimulus? Since bottom- up processes favor high saliency, detection of a low-salient target in the presence of highly salient distractors requires top-down attentional guidance. Previous studies have demonstrated that top-down attention can modulate perceptual processing and also that the control of attention is driven by frontoparietal regions. However, to date, there is no direct evidence on the cause and effect relationship between control regions and perceptual processing. Here, we report the first evidence demonstrating a neural circuit for the downregulation of salient distractors when a low-salient target is selected, combining brain imaging using functional magnetic resonance imaging with brain stimulation by transcranial magnetic stimulation. Using these combined techniques, we were able to identify a cause and effect relationship in the suppression of saliency, based on an interaction between the left intraparietal sulcus (IPS) and a region implicated in visual processing in our task (the left occipital pole). In particular, low-salient stimuli were selected by the left IPS suppressing early visual areas that would otherwise respond to a high-saliency distractor in the task. Apart from providing a first documentation of the neural circuit supporting selection by saliency, these data can be critical for understanding the underlying causes of problems in ignoring irrelevant salience that are found in both acquired and neurodevelopmental disorders (e.g., attention deficit/hyperactivity disorder or autism).
\end{abstract}

\section{Introduction}

Imagine searching for a dim target in a room where bright lights flash on and off. How do we ignore looking at the salient, bright lights and guide our attention to the low-saliency target? How do we accomplish this perceptual variant of ignoring the elephant in the room? Selection of the target under these circumstances depends on top-down guidance of attention away from highsaliency distractors favored by bottom-up processes. Previous studies have shown that attentional guidance can modulate perceptual processing of visual information in the brain of both monkeys (Moran and Desimone, 1985; Chelazzi et al., 1998, 2001) and humans (Kastner et al., 1998; Kastner and Ungerleider, 2001; Beck and Kastner, 2005; Sylvester et al., 2008). Attention to a particular location in the visual field activates retinotopic visual cortex (Kastner et al., 1999), whereas signals representing other locations are suppressed (Smith et al., 2000; Sylvester et al., 2008). Similarly, when a target is specified by a nonspatial attribute, such as object category (e.g., a face), activity in specialized extrastriate areas (e.g., the fusiform face area) is boosted (O'Craven et al., 1999; Serences et al., 2004; Gazzaley et al., 2005). Attention-based responses are also found in other more anterior brain regions, which are thought to provide control signals to regulate percep-

\footnotetext{
Received Jan. 15, 2010; revised Feb. 25, 2010; accepted March 22, 2010.

This work was supported by research grants from the Medical Research Council (United Kingdom) and the Biotechnology and Biological Sciences Research Council (United Kingdom).

Correspondence should be addressed to Carmel Mevorach, Behavioural Brain Sciences Centre, School of Psychology, University of Birmingham, Edgbaston, Birmingham B15 2TT, United Kingdom. E-mail: c.mevorach@ bham.ac.uk.

D0I:10.1523/JNEUROSCI.0241-10.2010

Copyright $@ 2010$ the authors $\quad 0270-6474 / 10 / 306072-08 \$ 15.00 / 0$
}

tual processing (Desimone and Duncan, 1995). In particular, many studies have linked both parietal [e.g., the intraparietal sulcus (IPS)] and frontal [such as the frontal eye fields (FEFs)] brain regions to top-down attentional control (Corbetta and Shulman, 2002; Serences and Yantis, 2006). However, imaging studies provide essentially correlatory evidence, and they do not demonstrate a causal link between attentional control processes in frontoparietal regions and implementation sites in perceptual processing regions. Such evidence requires direct manipulation of a brain region [e.g., through the neurodisruptive technique of transcranial magnetic stimulation (TMS) (Walsh and Cowey, 2000)]. In the present study, we used TMS coupled with functional brain imaging [functional magnetic resonance imaging (fMRI)] to investigate the causal relationship between attentional control areas (here, the IPS) and perceptual sites (occipital pole) underlying nonspatial attentional selection based on the relative saliency of the target and distractors.

In a series of recent studies (Mevorach et al., 2006a,b, 2009a,b; Hodsoll et al., 2009), we identified a lateralized top-down system for nonspatial selection by saliency. TMS applied to the left IPS immediately before a target display disrupts the selection of lowsaliency items and increases interference from high-saliency distractors (regardless of the visual field). This suggests that the left IPS is engaged in a preparatory manner, ostensibly to suppress the salient distractor and facilitate selection of the low-salient target. The role of the left IPS is specific to selection based on salience and not to task difficulty per se (Mevorach et al., 2009a). Here, we applied TMS over the left and right IPS to ask what effect these regions have on blood oxygen level-dependent (BOLD) activity in distal brain regions dealing with perceptual processing, 
focusing on conditions in which a high-saliency distractor must be ignored and a low-saliency target selected. In addition, we confirmed the proposed effects of the IPS on these distal regions by assessing effects of TMS on a distal region modulated by IPS activity.

\section{Materials and Methods}

In experiment 1, 12 participants performed two sessions of a global/local task [with the displays presented centrally, similar to the study by Mevorach et al. (2009b)] (see Fig. 1a) while functional MRI was acquired. Each session had two runs of the task, and in between 20 min of off-line TMS (Mevorach et al., 2006b) was applied either to the left or right IPS. This design enabled us to measure the effect of TMS-driven perturbation of a brain region with fMRI. Thus, we were able to identify activity changes in brain regions other than the stimulated area, but that can be directly attributed to TMS interference of IPS function. In experiment 2, TMS was applied directly over early visual cortex (based on the findings from experiment 1 ) to verify the contribution of this site to suppression of saliency.

\section{Experiment 1: combined off-line TMS and fMRI}

To assess the direct effect of the IPS on other, remote brain regions when stimuli with relatively high or low salience had to be selected, we combined off-line TMS over the left and right IPS with functional brain imaging (supplemental Fig. 1, available at www.jneurosci.org as supplemental material). Participants performed a global/local task with hierarchical letters in which we varied orthogonally the level of the stimulus (global or local) and the saliency of that level (high or low salience). This meant that any differential response to saliency was not confounded with the level of the stimulus. A previously identified cluster in the left IPS (Mevorach et al., 2009a) was used to guide TMS coil positioning. We verified the role of this imported cluster in the present study both using a region of interest (ROI) analysis (extracting the BOLD signal change in this region) and by contrasting whole-brain activity for conditions in which salient targets had to be selected and in which salient distractors had to be ignored. We then conducted a psychophysiological interaction (PPI) (see below) analysis to identify other remote brain areas that may be linked to the left or right IPS (without the intrusion of parietal TMS). To assess potential changes in connectivity after parietal TMS, we also conducted a similar PPI analyses on the post-TMS scans. Finally, we assessed the BOLD signal change in the potentially linked regions found in the PPI analysis as a function of left and right parietal TMS to ascertain the cause and effect relationship between the parietal cortex and these other remote areas.

\section{Method}

Participants. A group of 12 healthy participants (4 males; mean age, 30; all right-handed) with normal or corrected-to-normal vision took part. Where necessary, participants were fitted with MRI-compatible goggles replacing their glasses. The study was approved by the institutional ethics committee and conformed with the Declaration of Helsinki. All participants gave informed consent after the nature and possible consequences of the study were explained.

Stimuli. Two sets of displays were used in the global/local task to represent high global saliency and high local saliency and were presented on a black background. Stimuli were orthogonal compounds of the letters " $\mathrm{H}$ " and "S." For the condition with relatively high local saliency, each compound contained both red and white local letters (see Fig. 1a). Each local letter subtended $1.34 \times 1.76^{\circ}$ of visual angle (in width and height, respectively), and the global letter subtended $6.7 \times 10.81^{\circ}$ of visual angle (in width and height, respectively). The interelement distance was $0.46^{\circ}$. In the condition with relatively high global saliency, the compound letters were composed of the letters $\mathrm{H}$ and $\mathrm{S}$, and all the local letters were red. Each local letter subtended $1.34 \times 1.76^{\circ}$ of visual angle (in width and height, respectively), and the global letter subtended $5.83 \times 9.22^{\circ}$ of visual angle (in width and height, respectively). The interelement distance was $0.15^{\circ}$. These letters underwent a blur procedure in PaintShop Pro 7.0 with factor of 7 . The compound letters appeared centrally at one of two possible locations at $1.3^{\circ}$ above or below fixation along the vertical midline, which was done to ensure that participants performed the task under conditions encouraging diffuse attention (Grice et al., 1983).

\section{Experimental procedure}

Stimuli were projected onto a screen $\sim 620 \mathrm{~mm}$ from the participant's eyes and viewed by a mirror mounted on the head coil. On half of the trials, the compound figures consisted of the same global and local elements (congruent trials), and on the other half there were different global and local elements (incongruent trials). Each run of the task (a scan) included four different types of blocks (each $30 \mathrm{~s}$ ): global target under global-salient displays, global target under local-salient displays, local target under global-salient displays, and local target under local-salient displays. Each block type consisted of eight trials and was repeated three times in each run ( 12 blocks overall in each run). The main constraint on the number of repetitions was primarily attributable to the limited time in which the effects of off-line repetitive TMS (rTMS) last (i.e., the scan had to be finished within $10 \mathrm{~min}$ ). A written instruction ("global task" or "local task") appeared at the center of the screen $2 \mathrm{~s}$ before the beginning of each block. The blocks appeared in random order (different for each participant) separated by $16 \mathrm{~s}$ of fixation.

Each experimental trial started with the presentation of the fixation cross for $2000 \mathrm{~ms}$ followed by the target compound letter, presented for $150 \mathrm{~ms}$, followed by a $1850 \mathrm{~ms}$ response interval. Participants were then required to make a speeded response to the identity of the letter on the target level (H or S) by pressing one of two response keys on a keypad held in the right hand. Before the scanning sessions, participants completed a practice run outside the scanner consisting of four eight-trial blocks. Each participant participated in two sessions, which were performed on different days. A session comprised two runs of the task in the scanner interspersed with 20 min of TMS (see below) outside the scanner so that each participant performed the scanning sessions with both left and right IPS stimulation (within subject design). The average time from the end of the TMS train to the start of image acquisition in the post-TMS run was $\sim 3.5 \mathrm{~min}$, whereas a complete run of the task in the scanner took $10 \mathrm{~min}$.

TMS. A $70 \mathrm{~mm}$ figure-of-eight coil connected to a MagStim Rapid ${ }^{2}$ stimulator (MagStim) was positioned over two possible sites (either over the left or the right parietal cortex). The left parietal site was based on a left IPS cluster found in a previous fMRI study (Mevorach et al., 2009a) using a similar global/local task. This study failed to show right parietal activity linked to selection by saliency, so the right parietal site was used here as a control and was identified by flipping the left IPS cluster across the midline. Coil position was identified using the Brainsight TMS-MRI coregistration system (Rogue Research). The group cluster of left IPS activation (and its flipped counterpart) was transformed into the individual space and superimposed on each participant's high-resolution T1-weighted MR image. The coil was kept tangential to the head with the handle pointed posteriorly. Stimulation was given at $1 \mathrm{~Hz}$ with the intensity set to $10 \%$ below the individual motor threshold. Individual motor thresholds were established before the actual rTMS run, defined as the lowest stimulation intensity applied over the primary motor cortex to produce consistent, visible (in at least 7 of 10 repetitions) muscle twitches of the index finger (average motor threshold across participants: 60\%). This corresponds with a previous study (Mevorach et al., 2006b) in which the intensity of off-line stimulation over the parietal cortex was based on each participant's motor threshold. The order of stimulation sites (left or right IPS) was counterbalanced across participants. The rTMS train frequency, intensity, and duration were well within safe limits (Wassermann, 1998). After the completion of the TMS train, participants were immediately returned to the scanner room for the post-TMS scan.

fMRI imaging parameters. Images were acquired on a Philips 3T Achieva using the SENSE head coil. Functional images were acquired using an echo planar sequence [echo planar imaging (EPI)] with 33 transverse $3 \mathrm{~mm}$ slices [voxel size, $3 \times 3 \times 3 \mathrm{~mm}$; repetition time (TR), $2000 \mathrm{~ms}$; echo time (TE), $35 \mathrm{~ms}$; flip angle, $87^{\circ}$; field of view, $240 \mathrm{~mm}^{2}$; sense factor, $2 ; 300$ volumes in each scan]. Five dummy scans were acquired before data recording. A high-resolution T1-weighted anatomical scan was also acquired in the first run of each session with 175 sagittal slices, resolution of $1 \times 1 \times 1 \mathrm{~mm}$ (TR, 8.4; TE, 38; flip angle, $8^{\circ}$ ). 
fMRI preprocessing and analysis. The analysis was performed using FEAT (FMRI Expert Analysis Tool), version 5.4, part of FSL [Functional Magnetic Resonance Imaging of the Brain (FMRIB) Software Library; www.fmrib.ox.ac.uk/fsl]. Time series statistical analysis was performed using FILM with local autocorrelation correction (Woolrich et al., 2001). The first six volumes of each scan $(12 \mathrm{~s})$ were removed to reduce the transient effects of magnetic saturation and to allow the hemodynamic response to stabilize. Individual scans were preprocessed before additional analysis. Slice-timing correction (using Fourier space time series phase shifting) was applied. Movements of a participant's head were corrected for using MCFLIRT (Jenkinson et al., 2002). The average head movement by participants was low $(0.24 \mathrm{~mm}$; range, $0.09-0.58 \mathrm{~mm})$. The skull and other nonbrain matter were removed using BET (Smith, 2002). Images were then spatially smoothed using an isotropic Gaussian kernel of full width at half-maximum of $5 \mathrm{~mm}$ and all volumes within a scan were mean-based intensity normalized using the grand mean for the time series. Signals within the scan were then high-pass temporal filtered (Gaussian-weighted line spread function straight line fitting, with $\sigma=$ 90 s). Each participants' whole-head EPI image was registered to their individual (brain-extracted) structural image. Individual scans were registered to high-resolution standard images in Montreal Neurological Institute space using FLIRT (Jenkinson and Smith, 2001; Jenkinson et al., 2002). Four different types of blocks (global target/global salient, local target/local salient, global target/local salient, and local target/global salient) were used in a block design. Effects of saliency were assessed by comparing target-salient blocks (including the global-target/global-salient and local-target/local-salient experimental blocks) with distractorsalient blocks (including the global-target/local-salient and local-target/ global-salient experimental blocks). In addition, we also contrasted activity for all experimental blocks compared with rest to identify the visual areas implicated in task performance (before the application of parietal TMS).

ROI analysis. Two types of ROI analyses were conducted. In the first, a left IPS cluster was selected from our previous study with a similar global/ local task, which represents activity related to selection when distractors are salient (given the time period between the pre-TMS scans and the application of TMS, plus the relatively brevity of the scans, critical regions of the IPS could not be derived for the pre-TMS scan for each individual, to then target TMS). The time series in this cluster were extracted for each participant and we used PPI analysis (see below) to identify other brain regions showing correlated activity in the distractorsalient condition. Those time series were extracted initially from the (two) pre-TMS scans with the aim of identifying areas linked to the left IPS before TMS had any effect. Using this ROI analysis, we also verified the modulation of the left IPS cluster according to the saliency of the target and distractors or the level of form (global or local) participants responded to. Extraction of time series in these clusters was then also performed for the post-TMS scans to feed into PPI analysis of the postTMS scans, to assess connectivity changes after TMS.

A second ROI analysis was conducted with the occipital cluster identified in the PPI analysis of the pre-TMS scans to assess any direct modulation of its activity after left or right parietal TMS. This large cluster was divided into two smaller ones by simply bisecting it along the midline, resulting in a left occipital pole cluster (1129 voxels) and a right cuneal cluster (1761 voxels). For each participant, the maximum percentage BOLD signal change was extracted in the pre- and post-TMS scans of each TMS site (left and right IPS). This was then fed through to a repeated-measures ANOVA with saliency (target salient vs distractor salient), task (global vs local), and scan (pre-TMS vs post-TMS) as within-subject factors. To verify the source of one of the significant interactions found, we ran another ANOVA in which the data were coded with the factors of display (global salient vs local salient), task (global vs local), and scan (pre-TMS vs post-TMS).

PPI analysis. To identify a priori brain regions linked to left IPS activity when salient distractors had to be ignored, we conducted a psychophysiological interaction analysis on the two pre-TMS scans of each participant (Friston et al., 1997). For each pre-TMS scan, the extracted time series of the left and right IPS clusters were used to build two new models including the psychological regressor (distractor salient - target salient), a physiological regressor (the time series of the left or right IPS), and a PPI regressor (psychological by physiological). The results of these PPI analyses were averaged first within each participant over the two preTMS scans. Higher-level, group, analysis was performed using FLAME (FMRIB Local Analysis of Mixed Effects) (Beckmann et al., 2003; Woolrich et al., 2004). $Z$ (Gaussianised T/F) statistic images were thresholded using clusters determined by $Z>2.8$ and a (corrected) cluster significance threshold of $p=0.05$ (Worsley et al., 1992). A similar analysis was performed for the post-TMS scans (this time separately for post-TMS of left IPS and post-TMS of right IPS) to test whether connectivity was affected by TMS to the parietal cortices.

\section{Experiment 2: on-line TMS over the left occipital pole}

The findings of experiment 1 indicated that a region in the left occipital pole, activated in the task, was modulated by the left IPS when salient distractors had to be ignored. Specifically, interference with the left IPS resulted in an increase in activity in the occipital region only in the distractor-salient condition. To provide a direct verification of this finding in experiment 2, we applied TMS directly over the left occipital pole region (supplemental Fig. 2, available at www.jneurosci.org as supplemental material), using a protocol that has previously been shown to reduce the BOLD signal in the stimulated area (Sack et al., 2007). If the left IPS has an inhibitory effect on the occipital pole region when salient distractors need to be ignored, then direct disruption of that brain region (using TMS) should facilitate performance in the distractor-salient condition.

\section{Method}

Participants. Twelve healthy right-handed participants drawn from the local student population at the University of Birmingham gave written informed consent to participate in the study after the nature and possible consequences of the study were explained to them and were naive to its purpose. The study was conducted in accordance with the Declaration of Helsinki, and the methodology was approved by the local ethics committee.

TMS. A $70 \mathrm{~mm}$ figure-of-eight coil connected to a MagStim Rapid stimulator (MagStim) was positioned over either $\mathrm{Cz}$ (the control site: 10-20 EEG coordinate system) or the left occipital pole. For the latter, the position of the coil was identified using the Brainsight TMS-MRI coregistration system (Rogue Research). The group cluster for the occipital pole site taken from experiment 1 was transformed into individual space and superimposed on each participant's high-resolution T1weighted MR image. The coil was kept tangential to the head with the handle pointed downward. Each trial consisted of five TMS pulses presented at a frequency of $10 \mathrm{~Hz}$ and at $60 \%$ of the maximum output of the stimulator with the first pulse given $100 \mathrm{~ms}$ before the onset of the visual information. Here, we followed previous studies and used a constant TMS level across participants (Mevorach et al., 2009b). This TMS protocol has recently been shown to reduce the BOLD signal in the stimulated region in a concurrent TMS/fMRI study (Sack et al., 2007). Stimulation was started before the onset of the visual targets following our previous work in which left posterior parietal cortex stimulation was shown to affect performance when given before the display (Mevorach et al., $2009 \mathrm{~b}$ ). This previous result suggested that modulatory effects from the left IPS take place before the onset of a visual display. The rTMS train frequency, intensity, and duration were well within safety limits (Wassermann, 1998).

Stimuli. The stimuli were presented on a 17 inch monitor $(1024 \times 768$ pixels) of a Gateway personal computer. The viewing distance was $\sim 60$ $\mathrm{cm}$ so that each centimeter on the screen represented 0.96 degrees of visual angle. All the stimuli appeared against a black background. Two sets of displays were used to represent high global saliency and high local saliency. For the condition with relatively high local saliency, the compound stimuli were created from orthogonal combinations of the letters $\mathrm{H}$ and $\mathrm{S}$. Each compound contained both red and white local letters (see Fig. 1a). Each local letter subtended $1.34 \times 1.06^{\circ}$ of visual angle (in width and height, respectively), and the global letter subtended $8.26 \times 5.38^{\circ}$ of visual angle (in width and height, respectively). The interelement distance was $0.38^{\circ}$. In the condition with relatively high global saliency, the compound letters were again comprised of the letters $\mathrm{H}$ and $\mathrm{S}$, which 
were combined orthogonally at the local and global levels. All the local letters were red. Each local letter subtended $1.34 \times 1.06^{\circ}$ of visual angle (in width and height, respectively), and the global letter subtended $5.66 \times 4.51^{\circ}$ of visual angle (in width and height, respectively). The distance between the local elements was $0.96^{\circ}$. These letters underwent a blur procedure in PaintShop Pro 7.0 with factor of 7. The compound letters could appear at one of two possible locations at $1.3^{\circ}$ above or below fixation along the vertical midline. A white cross $\left(0.57^{\circ}\right)$ served as fixation and was presented in the center of the screen.

\section{Experimental procedure}

On different blocks of 40 trials, participants were asked to identify the global or the local elements of the compound letter while ignoring information on the other level. On half of the trials, the compound figures consisted of the same global and local elements (congruent trials), and on the other half there were different global and local elements (incongruent trials). Each trial began with the presentation of a fixation cross for 1500 $\mathrm{ms}$. After a $200 \mathrm{~ms}$ interval, the target compound letter appeared for 50 ms. Participants were required to make a speeded response to the identity of the letter on the target level ( $\mathrm{H}$ or $\mathrm{S}$ ) by pressing one of two keyboard keys $(\mathrm{k}$ and $\mathrm{m})$ using their right hand. After the key press, the next trial began. In addition, a sequence of five TMS pulses (100 ms apart) was given starting $100 \mathrm{~ms}$ before the onset of the target compound letter either over $\mathrm{Cz}$ or the left occipital pole. The order of the stimulation sites was randomized with stimulation at each site performed on a different day. Each run of the task included four blocks (two with "global" targets and two with "local" targets). A written instruction (global task or local task) appeared at the center of the screen $2 \mathrm{~s}$ before the beginning of each block. The first two blocks and the last two blocks of each run were both either with the global letter being more salient or with the local letters being more salient (the order was counterbalanced across subjects and runs).

\section{Results}

\section{Experiment 1: combined TMS/fMRI}

Behaviorally, there were main effects of saliency rather than interactions with congruency, similar to our previous behavioral data collected in an fMRI environment (Mevorach et al., 2009a) (it seems that differential effects of congruence on high- and low-saliency targets are relatively more fragile than main effects of saliency, although they have been found outside the scanner environment). Essentially, TMS to the left and right IPS differentially affected performance. The effects were primarily visible in the change in performance between pre-TMS and post-TMS runs (Fig. 1b). In general, performance improved across the two runs, as would be expected from a general practice effect (supplemental Fig. 3, available at www.jneurosci.org as supplemental material). However, left or right IPS TMS interacted with this general improvement (for a similar example, see Mevorach et al., 2005). A repeated-measures ANOVA on the response time (RT) differences (RT post-TMS - RT pre-TMS) with stimulation site (left vs right IPS) and saliency (target salient vs distractor salient) as within-subject factors revealed a two-way interaction $\left(F_{(1,11)}=\right.$ 7.665; $p<0.025$ ) (for a full analysis, see supplemental Fig. 3 and accompanying text, available at www.jneurosci.org as supplemental material). For TMS to the left IPS, there was a greater improvement over time (pre- vs post-TMS) when the target was salient $(-23 \mathrm{~ms})$ compared with when the distractor was salient $\left(-4 \mathrm{~ms} ; t_{(11)}=1.789, p=0.054\right)$. After TMS to right IPS, the improvement in RTs across sessions was larger when the distractor was salient $(-30 \mathrm{~ms})$ than when the target was salient $(5 \mathrm{~ms}$; $\left.t_{(11)}=3.297 ; p=0.004\right)$. Given the general improvement in RTs (similar to Mevorach et al., 2005), the effects of TMS to left and right IPS match our previous data (Mevorach et al., 2006b), in which left IPS disrupted performance with highly
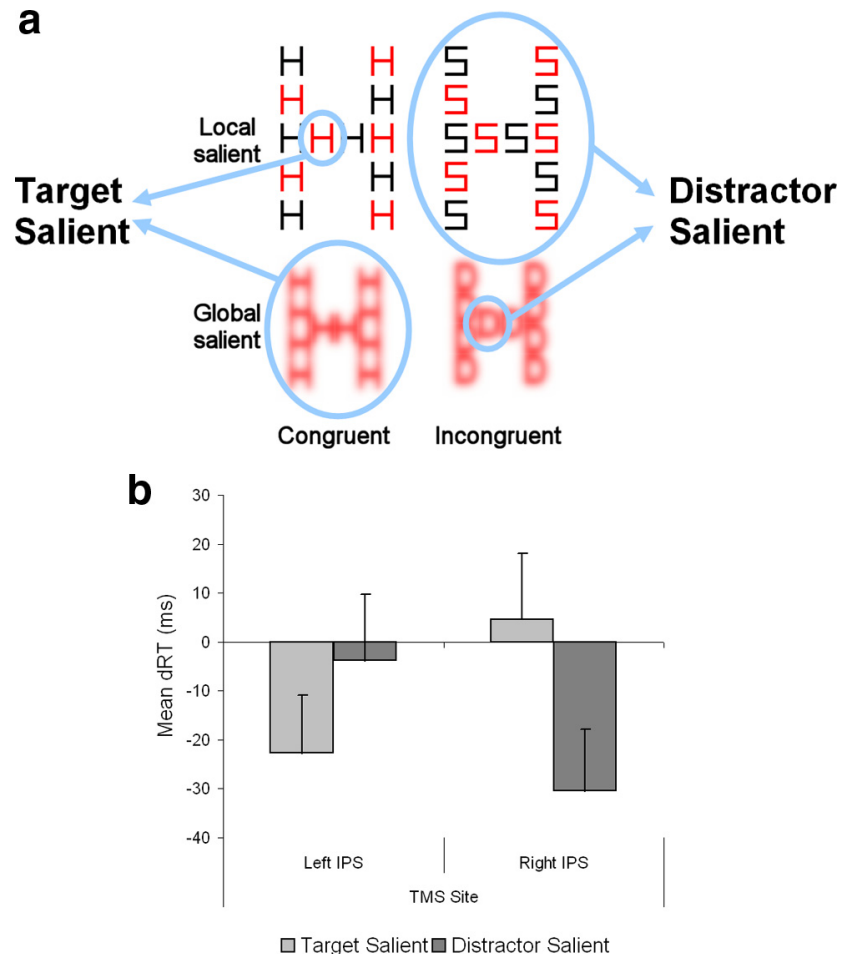

Figure 1. Example of the experimental task and behavioral results of experiment 1. $\boldsymbol{a}$, Two display sets were used, one with local saliency (top pair) and another with global saliency (bottom pair). On different blocks of trials, participants were asked to respond to either the local or the global aspect of the hierarchical letter. Responses for the local letter under conditions of local saliency were combined with responses for the global letter under conditions of global saliency to form a target-salient condition. Similarly, responses to the global letter under conditions of local saliency were combined with responses to the local letter under conditions of global saliency to form a distractor-salient condition. $\boldsymbol{b}$, The mean change in RTs from pre-TMS to post-TMS, recorded in the MR scanner. Negative values represent improvement from pre- to post-TMS runs, expected from a general practice effect. Error bars indicate SEM.

salient distractors, whereas right IPS disrupted performance with highly salient targets.

To verify the role of the left IPS cluster [taken from our previous study (Mevorach et al., 2009a)] at a neural level in the present study, the average peak BOLD signal change was extracted for this left IPS cluster in the four experimental blocks of the pre-TMS scans. A repeated-measures ANOVA was performed on the BOLD signal change with scan (pre-TMS left vs pre-TMS right), saliency (target salient vs distractor salient), and task (global vs local) as factors (for a list of the peak BOLD signal change in all conditions, see supplemental Table 1, available at www.jneurosci.org as supplemental material). In accordance with our previous findings, there was a main effect of saliency $\left(F_{(1,12)}=8.675 ; p<0.025\right)$. BOLD signal change in the left IPS was significantly larger in distractor-salient conditions $(0.85 \%)$ compared with target-salient conditions $(0.71 \%)$. There was recruitment of the left IPS when low-salient targets need to be selected and high-salient distractors ignored. No other significant main effects or interactions were found, including no significant effect for task $\left(F_{(1,11)}<1\right)$. There was, therefore, no evidence that the left IPS cluster was modulated by the level of form participants were identifying ( 0.76 and $0.79 \%$ BOLD signal change for local and global tasks, respectively). In addition, we conducted a whole-brain analysis to contrast conditions in which the distractor was more salient with conditions in which the target was more salient (supplemental Fig. 4, supplemental Table 2 and accompa- 


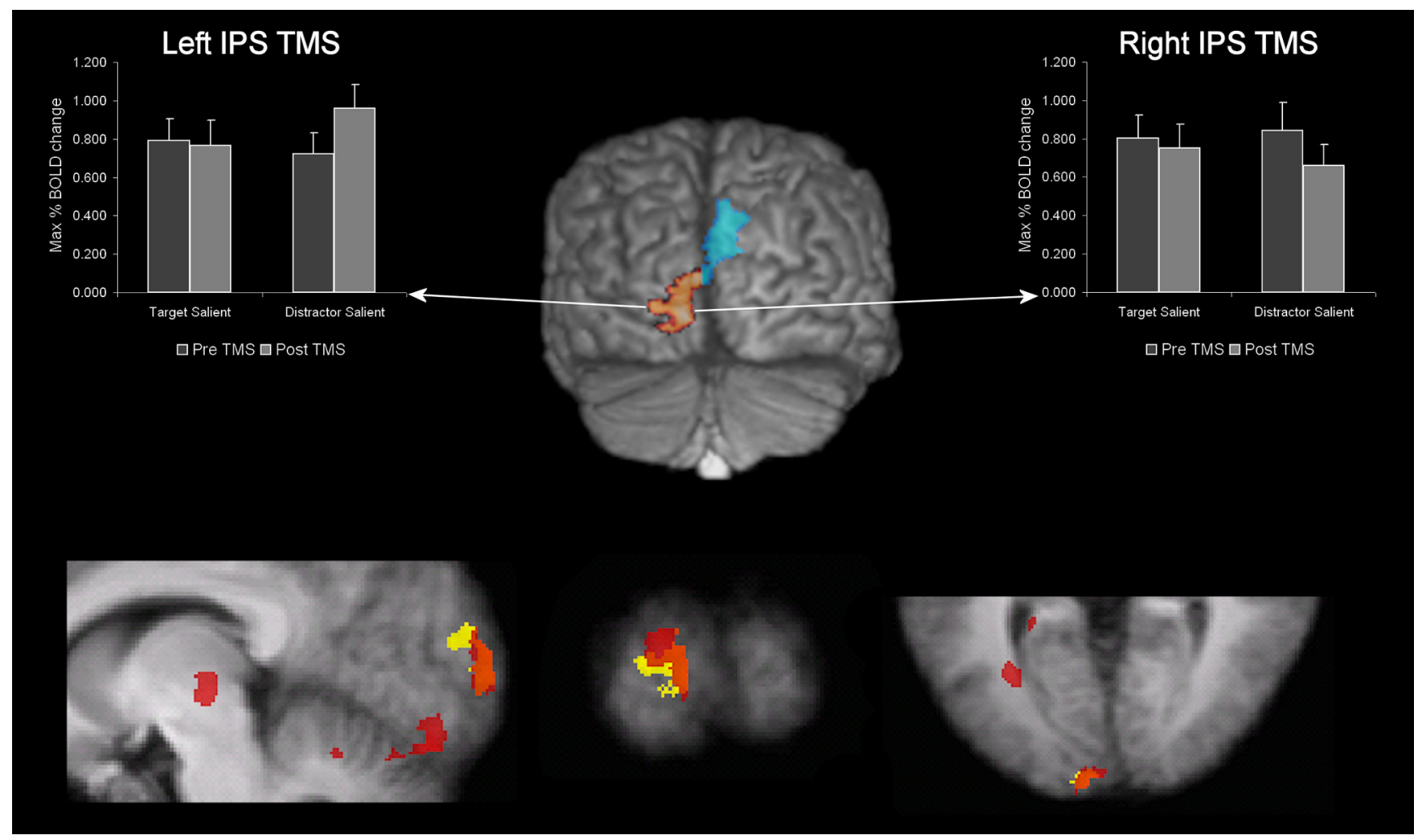

Figure 2. Significant activation related to the psychological by physiological component showing negative correlation with activity in the left IPS in distractor-salient conditions (center of figure; $n=12 ; p<0.05$, corrected). The group activation is superimposed on an individual T1-weighted structural image. The two subclusters are seen in orange (left occipital pole) and blue (right cuneus). At the bottom of the figure, activation map for the visual $>$ rest contrast of the pre-TMS scans in experiment 1 is shown. The group activation ( $n=12 ; p<0.05$, corrected) (for a full list of clusters, see supplemental Table 3, available at www.jneurosci.org as supplemental material) is superimposed (in red) on an averaged T1-weighted structural image showing the left occipital pole cluster (the orange cluster from the image at the center of the figure) in yellow. The overlap between the clusters is shown in orange. BOLD signal change in the left occipital pole cluster for the different saliency conditions before and after left and right IPS TMS is shown on the left and right of the figure, respectively. Here, we show the maximum BOLD signal change (denoted in percentage), averaged across all participants. Error bars denote interparticipant SE.

nying text, available at www.jneurosci.org as supplemental material). This comparison indicated a good overlap between the cluster exported from our previous fMRI study (Mevorach et al., 2009a) and parietal activation in the present study (supplemental Fig. 4, available at www.jneurosci.org as supplemental material), confirming that TMS was applied to an area implicated in selection by saliency for the current participants.

To assess TMS effects on brain activity, we identified areas putatively linked to the role of the left or right IPS in suppressing or selecting salient information. Two PPI analyses (Friston et al., 1997) were conducted on the pre-TMS scans, one with the left IPS cluster found in our previous study (Mevorach et al., 2009a) as the seed region (see Materials and Methods) and one with the right IPS cluster. The PPI analysis with the left IPS revealed a large occipital cluster expanding from left occipital pole to more superior right cuneus (Fig. 2a; supplemental Table 3, available at www.jneurosci.org as supplemental material), which was negatively correlated with activity in the left IPS when the distractor was more salient than the target. That is, an increase in left IPS activity in this condition was coupled with a decrease in activity in the occipital cluster, suggesting suppression of the occipital region by the left IPS when the salient distractor had to be ignored. In contrast, the PPI analysis with the right IPS revealed no significant coupling of this region with any other brain region.

To provide evidence for a causal interaction between left IPS and the occipital region, we analyzed the change in the occipital BOLD signal after TMS interference with the left and right IPS.
The occipital cluster was separated into two subclusters along the midline (effectively creating a left occipital pole cluster and a right superior cuneal cluster) (Fig. 2, middle). As the occipital pole may correspond with early visual cortex, we compared the peak voxel of this cluster $(-6,-102,3)$ with the cytoarchitectonic maps using the SPM Anatomy Toolbox (Eickhoff et al., 2005). This suggested high probability (90\%) for the peak voxel to correspond to left calcarine gyrus and Brodmann area 17 (supplemental Table 3, available at www.jneurosci.org as supplemental material), which normally shows high overlap with functional V1. To further verify the role of the left occipital pole cluster in visual representation of stimuli in the present task, we also compared activity over the whole brain during the visual blocks to rest, in the two pre-TMS scans (pooling left- and right-parietal TMS sessions). Supplemental Table 4 (available at www. jneurosci.org as supplemental material) lists the active clusters for this comparison. Activity in the occipital pole was lateralized to the left for this contrast and corresponded with the left occipital pole cluster found in the PPI analysis (Fig. 2, bottom). This lateralization may reflect factors such as our use of letter stimuli; regardless of this, the data indicate that the left occipital pole cluster identified in the PPI analysis corresponds well with the main region of visual cortical activity implicated in our task.

Next, the average peak BOLD signal change was extracted for each of the two occipital clusters for the different experimental blocks, for the pre- and post-TMS scans (separately, after left and right IPS). ROI analysis revealed two different patterns exhibited 
by the two subclusters. For the right cuneal cluster (supplemental Fig. 5a,b, available at www.jneurosci.org as supplemental material), repeated-measures ANOVAs with time (pre-TMS vs postTMS), saliency (target salient vs distractor salient), and task (global vs local) were performed for TMS to the left and right IPS separately. They revealed no significant change of the BOLD signal for any of the conditions, or any interactions, for either TMS site.

More importantly, for the left occipital pole region, left IPS stimulation resulted in a specific change of activity (Fig. 2, left; supplemental Table 5, available at www.jneurosci.org as supplemental material). A repeated-measures ANOVA with time (prevs post-TMS), saliency (target salient vs distractor salient), and task (global vs local) as within-subject factors revealed a two-way interaction $\left(F_{(1,11)}=5.699 ; p<0.05\right)$ of time and saliency. There was no significant difference before $(0.79 \%)$ and after $(0.76 \%)$ TMS to the left IPS in the target-salient condition $\left(t_{(11)}<1\right.$, NS). However, when the distractor was salient, activity after TMS $(0.96 \%)$ was significantly greater than before TMS $\left(0.72 \% ; t_{(11)}=\right.$ $3.463 ; p<0.005)$. In addition, there was also a significant interaction of task and saliency $\left(F_{(1,11)}=6.941 ; p<0.025\right)$. This interaction represents a main effect of display type [that is, whether the displays are blurred (as in the global-salient conditions) or sharp (as in the local-salient conditions)] (supplemental Fig. 6 and accompanying text, available at www.jneurosci.org as supplemental material). Stimulation of the right IPS only revealed a similar significant two-way interaction of task and saliency $\left(F_{(1,11)}=5.893 ; p<0.05\right)$. This interaction can also be attributed to an effect of display type (see above) (supplemental Fig. 6 and accompanying text, available at www.jneurosci.org as supplemental material). None of the interactions or main effects including the factor of time (pre- and post-TMS) was significant (Fig. 2, left).

The data indicate that parietal TMS modulated a distal brain region (the occipital pole). However, when we compared BOLD activity in the left and right IPS before and after stimulation, no significant change in activity was found (supplemental Table 6 and accompanying text, available at www.jneurosci.org as supplemental material). This lack of change in the BOLD signal fits with previous reports using a similar methodology (Rounis et al., 2006; O'Shea et al., 2007). This is in contrast with on-line $10 \mathrm{~Hz}$ stimulation, which has been shown to reduce BOLD signal change in the stimulated area (Sack et al., 2007). It is a matter of additional investigations to reveal the link between the neuronal effect of subthreshold off-line stimulation and the BOLD signal in the stimulated area.

Finally, we also conducted PPI analyses on the left and right IPS clusters after left and right parietal TMS to assess potential changes in connectivity, compared with when the same analysis was performed before the application of TMS. For both analyses, we found no significant activations (either in occipital areas or elsewhere). It should be noted, however, that this last PPI analysis involved only one scan (post left or right parietal TMS), whereas the PPI conducted on the pre-TMS scans was averaged over the two pre-TMS scans. The post-TMS PPI analysis may thus be underpowered compared with the pre-TMS data and that left IPS occipital pole coupling may exist but be undetected.

Together, these findings show a clear difference between the effects of left and right IPS stimulation on activity in the occipital cortex. Importantly, when salient distractors had to be ignored and less salient targets selected, disruption of activity in the left IPS resulted in increased activity in a left occipital pole region active in our identification task. On top of this TMS-induced change in activity, the left occipital pole was also shown to be driven by the physical characteristics of the visual information. Blurred displays (global salient) resulted in less BOLD activation than sharp displays (local-salient stimuli) (supplemental Fig. 6, available at www.jneurosci.org as supplemental material), but there was not modulation based on the level of form participants were required to report (global or local). The findings also indicate reduced coupling after TMS to the left IPS between the left IPS and the occipital pole region implicated in visual processing in our task.

This result verifies that there is an inhibitory relationship between the left IPS and the left occipital pole. Downregulation of the left occipital pole, by the left IPS, may prevent rapid orienting to the salient distractor, consequently improving selection of a low-saliency target. If this is indeed the case, then there should also be a selective benefit to performance in the distractor-salient condition if we mimic the inhibitory effect of left IPS, by applying TMS directly to the visual cortex. Such a test will also help exclude the possibility that the difference in BOLD signal found before and after TMS to the left IPS was a result of a general difference between those scans. This was tested in experiment 2 .

\section{Experiment 2: TMS suppression of the left occipital cluster}

We asked participants to perform the same global/local task as before while on-line TMS (5 pulses at $10 \mathrm{~Hz}$ ) was applied to disrupt activity in a control site $(\mathrm{Cz})$ and in the occipital pole region identified in experiment 1 (Fig. $3 a$ ). As predicted, TMS had a differential effect on performance in conditions with salient targets and distractors (relative to control site, $\mathrm{Cz}$ ) (Fig. 3b; supplemental Tables 9, 10, available at www.jneurosci.org as supplemental material) with the effect of congruency here generally replicating our previous behavioral studies conducted outside the fMRI environment (Mevorach et al., 2006a,b). A repeatedmeasures ANOVA was conducted on RTs with site (Cz vs left occipital pole), saliency (target-salient vs distractor-salient), task (global vs local), and congruency of the global/local letter (congruent vs incongruent) as within-subject factors. Critically, there was a significant interaction between site, saliency, and congruency $\left(F_{(1,11)}=5.988 ; p<0.05\right.$ ) (for the full report of the RT as well as accuracy ANOVAs, see supplemental Fig. 7 and accompanying text, available at www.jneurosci.org as supplemental material). For the target-salient condition there was no difference in the interference effects (incongruent RTs - congruent RTs) at each stimulation site ( $40 \mathrm{vs} 30 \mathrm{~ms}$ for left occipital and Cz, respectively; $\left.t_{(11)}<1\right)$. For the distractor-salient condition, interference was significantly reduced after TMS over the left occipital pole (56 ms) compared with TMS over $\mathrm{Cz}\left(76 \mathrm{~ms} ; t_{(11)}=2.84 ; p<\right.$ $0.01)$. Thus, disrupting activity in the left occipital pole made it easier to ignore highly salient but distracting information when the target was low in salience. We note also that overall performance was not affected by left occipital pole stimulation $\left(F_{(1,11)}<1\right.$ for the main effect of TMS) (supplemental Fig. 7, available at www.jneurosci.org as supplemental material). In particular, there was no difference in overall performance in the distractorsalient condition for the two stimulation sites ( 514 and $513 \mathrm{~ms}$ for left occipital and $\mathrm{Cz}$ stimulation, respectively; $\left.t_{(11)}<1\right)$. Therefore, the reduction in the congruency effect for the distractorsalient condition, after occipital pole stimulation, cannot be an artifact of a general reduction in perceptual processing because of stimulation applied over the visual cortex. 

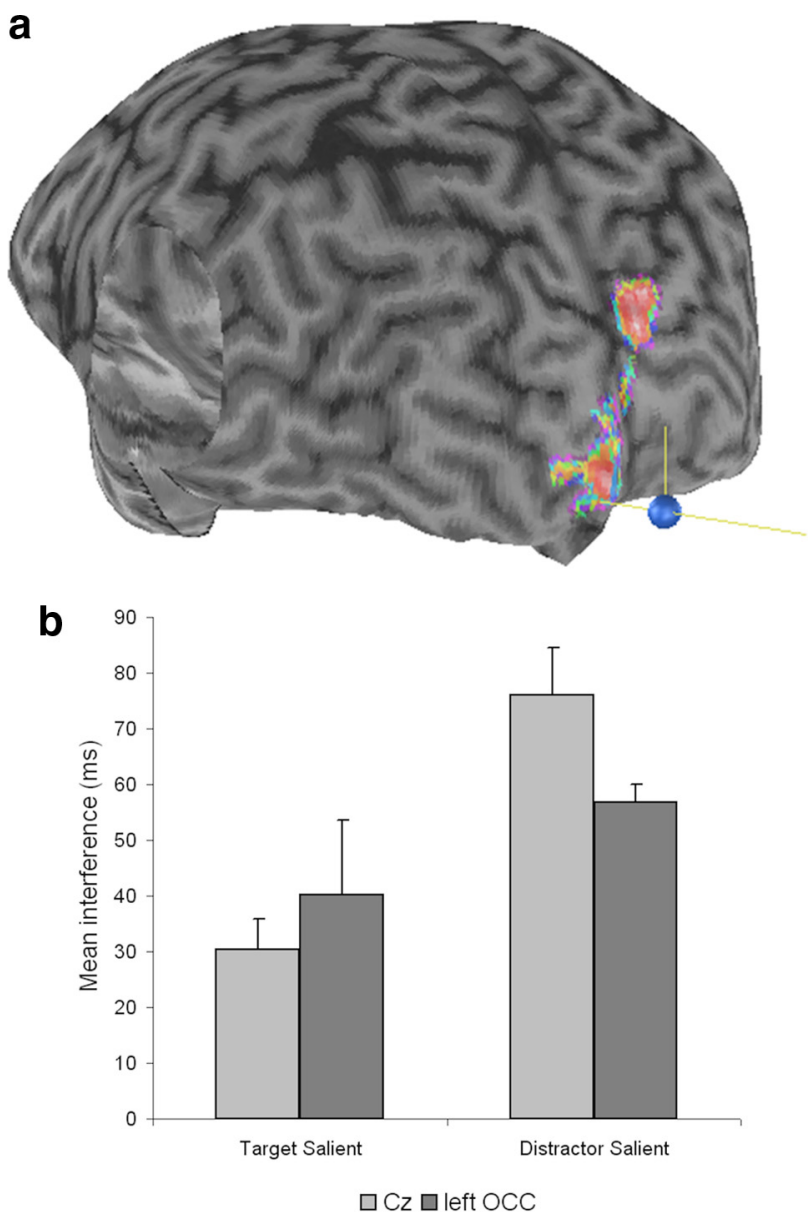

Figure 3. $a$, Example of localization of the TMS coil over the occipital pole region of an individual participant (using the Brainsight system) in experiment 2. The picture shows the group PPI cluster found in experiment 1 transformed and superimposed in the individual space. The blue sphere represents the coil position with the yellow axis representing coil orientation. The coil was targeted at the voxel showing peak activity in the left occipital pole region, which was actually slightly deeper than the surface activation shown. $\boldsymbol{b}$, Mean interference (congruency) effects in RTs (+SEM) after TMS over left occipital or Cz sites.

\section{Discussion}

To date, the conjecture that frontoparietal regions serve as control sites sending signals to perceptual areas to implement attentional selection is mainly based on correlatory brain imaging data in humans. Applying causal time series analysis to fMRI BOLD data has supported the argument for functional connectivity (e.g., Granger causality analysis) (Bressler et al., 2008), but only direct manipulation of a brain region can provide definitive evidence for a cause and effect relationship. Indeed, the efficacy of using TMS (including in combination with imaging techniques) to show causal influences of one brain region on another has been demonstrated recently where direct stimulation over the FEF and IPS has shown to modify excitability (Silvanto et al., 2006, 2009) as well as the BOLD signal in visual cortex (Ruff et al., 2006, 2008). These previous investigations indicate the presence of neural connectivity that may implement the link between attention control areas and their potential effect over perceptual areas.

The present data complement those previous investigations by providing direct evidence that the ability to ignore salient visual distractors is contingent on a neural circuit involving both the left IPS (control site) and occipital pole (implementation site). Critically, our use of a "perturb and measure" approach enables us to make causal inferences about the interplay between these brain areas. We found that activity in the left IPS was correlated negatively with activation of the left occipital pole, when salient distractors had to be ignored in our task. A causal link between activity in these two regions was then established using TMS. Suppressive TMS to the left IPS increased activity in the occipital pole in the distractor-salient condition. This is consistent with TMS to left IPS releasing the suppressive effect of that region on the occipital pole, when salient distractors had to be ignored. A causal role for the early visual regions at the occipital pole was then demonstrated by showing that TMS to this region selectively decreased interference from salient distractors. We suggest that the IPS acts to downregulate an occipital response when salient distractors have to be ignored, which in turn prevents a bottom-up orienting response to salience. This is mimicked by direct application of TMS to the occipital pole.

One aspect of the current study is that the saliency of the target was varied orthogonally with whether the target was located at the local or global level of the stimulus. Thus, the effects of downregulating the occipital pole cannot be attributed to a reduced response to either local or global aspects of a stimulus. In accord with this conjecture, it was shown that the occipital pole was not modulated by the specific task participants were performing (report global or local target) but rather by the general physical characteristics of the display. Experiment 2 also showed that there was not a general decrease in visual processing after TMS, but the effect was specific to when highly salient distractors had to be ignored.

Although our current data highlight an interaction between the left IPS and the left occipital pole, for suppressing saliency, it should be noted that the left occipital region was activated in our task under all conditions. Our data do not indicate that the left occipital regions is always implicated in suppressive processing, but only that suppression acts on a region that is normally strongly implicated in perceptual processing for the stimuli in the task.

How can an effect specific to high-saliency stimuli arise? One possibility is that there is a nonlinear increase in neural firing as stimuli increase in saliency. If TMS introduces a proportional decrease in activation, then the reduction will be greatest for highly salient stimuli. An alternative view is that on-line TMS reduces activity in hyperactive subpopulations of neurons in a particular brain region, without affecting the subpopulation of hypoactive neurons (Silvanto et al., 2008). If the hyperactive neurons respond to highly salient inputs, then the neural response to saliency will reduce when the hyperactive neurons are "dampened." In either case, we need also to ask why this affected performance only when distractors were salient and not targets. To account for this, we propose that effects of reducing the neural response to visual salience may be offset to some degree when the target is salient and so matches the top-down set. Nevertheless, we note that there was a trend for overall responses toward highsaliency targets being affected by left occipital TMS (supplemental Fig. 7, available at www.jneurosci.org as supplemental material), which may hint at a degraded representation of saliency, even for targets. The important point, however, is that this selective benefit is predicted by our proposed functional connectivity in an IPS-occipital pole circuit in the left hemisphere, where downregulation of the occipital pole by the IPS underlies an ability to ignore salient distraction.

The present findings also suggest that problems in suppressing irrelevant but salient stimuli, found after brain lesions (Mevorach et al., 2006b), may be linked to various neuroanatomical loci 
including the following: abnormalities in the left IPS itself, decreases in effective connectivity between the left IPS and visual cortex, or even heightened activity in the occipital pole. It will be important to trace the exact cause of any problem in ignoring irrelevant salience that may be found in both acquired and neurodevelopmental disorders (e.g., attention deficit/hyperactivity disorder or autism).

\section{References}

Beck DM, Kastner S (2005) Stimulus context modulates competition in human extrastriate cortex. Nat Neurosci 8:1110-1116.

Beckmann CF, Jenkinson M, Smith SM (2003) General multi-level linear modeling for group analysis in FMRI. Neuroimage 20:1052-1063.

Bressler SL, Tang W, Sylvester CM, Shulman GL, Corbetta M (2008) Topdown control of human visual cortex by frontal and parietal cortex in anticipatory visual spatial attention. J Neurosci 28:10056-10061.

Chelazzi L, Duncan J, Miller EK, Desimone R (1998) Responses of neurons in inferior temporal cortex during memory-guided visual search. J Neurophysiol 80:2918-2940.

Chelazzi L, Miller EK, Duncan J, Desimone R (2001) Responses of neurons in macaque area V4 during memory-guided visual search. Cereb Cortex 11:761-772.

Corbetta M, Shulman GL (2002) Control of goal-directed and stimulusdriven attention in the brain. Nat Rev Neurosci 3:201-215.

Desimone R, Duncan J (1995) Neural mechanisms of selective visual attention. Annu Rev Neurosci 18:193-222.

Eickhoff SB, Stephan KE, Mohlberg H, Grefkes C, Fink GR, Amunts K, Zilles K (2005) A new SPM toolbox for combining probabilistic cytoarchitectonic maps and functional imaging data. Neuroimage 25:1325-1335.

Friston KJ, Buechel C, Fink GR, Morris J, Rolls E, Dolan RJ (1997) Psychophysiological and modulatory interactions in neuroimaging. Neuroimage 6:218-229.

Gazzaley A, Cooney JW, McEvoy K, Knight RT, D’Esposito M (2005) Topdown enhancement and suppression of the magnitude and speed of neural activity. J Cogn Neurosci 17:507-517.

Grice GR, Canham L, Boroughs JM (1983) Forest before trees? It depends where you look. Percept Psychophys 33:121-128.

Hodsoll J, Mevorach C, Humphreys GW (2009) Driven to less distraction: rTMS of the right parietal cortex reduces attentional capture in visual search. Cereb Cortex 19:106-114.

Jenkinson M, Smith S (2001) A global optimization method for robust affine registration of brain images. Med Image Anal 5:143-156.

Jenkinson M, Bannister P, Brady M, Smith S (2002) Improved optimization of the robust and accurate linear registration and motion correction of brain images. Neuroimage 17:825-841.

Kastner S, Ungerleider LG (2001) The neural basis of biased competition in human visual cortex. Neuropsychologia 39:1263-1276.

Kastner S, De Weerd P, Desimone R, Ungerleider LG (1998) Mechanisms of directed attention in the human extrastriate cortex as revealed by functional MRI. Science 282:108-111.

Kastner S, Pinsk MA, De Weerd P, Desimone R, Ungerleider LG (1999) Increased activity in human visual cortex during directed attention in the absence of visual stimulation. Neuron 22:751-761.

Mevorach C, Humphreys GW, Shalev L (2005) Attending to local form while ignoring global aspects depends on handedness: evidence from TMS. Nat Neurosci 8:276-277.

Mevorach C, Humphreys GW, Shalev L (2006a) Effects of saliency, not global dominance, in patients with left parietal damage. Neuropsychologia 44:307-319.

Mevorach C, Humphreys GW, Shalev L (2006b) Opposite biases in salience-based selection for the left and right posterior parietal cortex. Nat Neurosci 9:740-742.

Mevorach C, Shalev L, Allen HA, Humphreys GW (2009a) The left in- trapariatel sulcus modulates the selection of low salient stimuli. J Cogn Neurosci 21:303-315.

Mevorach C, Humphreys GW, Shalev L (2009b) Reflexive and preparatory selection and suppression of salient information in the right and left posterior parietal cortex. J Cogn Neurosci 21:1204-1214.

Moran J, Desimone R (1985) Selective attention gates visual processing in the extrastriate cortex. Science 229:782-784.

O'Craven KM, Downing PE, Kanwisher N (1999) fMRI evidence for objects as the units of attentional selection. Nature 401:584-587.

O'Shea J, Johansen-Berg H, Trief D, Göbel S, Rushworth MF (2007) Functionally specific reorganization in human premotor cortex. Neuron 54:479-490.

Rounis E, Stephan KE, Lee L, Siebner HR, Pesenti A, Friston KJ, Rothwell JC, Frackowiak RS (2006) Acute changes in frontoparietal activity after repetitive transcranial magnetic stimulation over the dorsolateral prefrontal cortex in a cued reaction time task. J Neurosci 26:9629-9638.

Ruff CC, Blankenburg F, Bjoertomt O, Bestmann S, Freeman E, Haynes JD, Rees G, Josephs O, Deichmann R, Driver J (2006) Concurrent TMS/ fMRI and psychophysics reveal frontal influences on human retinotopic visual cortex. Curr Biol 16:1479-1488.

Ruff CC, Bestmann S, Blankenburg F, Bjoertomt O, Josephs O, Weiskopf N, Deichmann R, Driver J (2008) Distinct causal influences of parietal versus frontal areas on human visual cortex: evidence from concurrent TMS/ fMRI. Cereb Cortex 18:817-827.

Sack AT, Kohler A, Bestmann S, Linden DE, Dechent P, Goebel R, Baudewig J (2007) Imaging the brain activity changes underlying impaired visuospatial judgments: simultaneous FMRI, TMS, and behavioral studies. Cereb Cortex 17:2841-2852.

Serences JT, Yantis S (2006) Selective visual attention and perceptual coherence. Trends Cogn Sci 10:38-45.

Serences JT, Schwarzbach J, Courtney SM, Golay X, Yantis S (2004) Control of object-based attention in human cortex. Cereb Cortex 14:1346-1357.

Silvanto J, Lavie N, Walsh V (2006) Stimulation of the human frontal eye fields modulates the sensitivity of the extrastriate visual cortex. J Neurophysiol 96:941-945.

Silvanto J, Cattaneo Z, Battelli L, Pascual-Leone A (2008) Baseline cortical excitability determines whether TMS disrupts or facilitates behavior. J Neurophysiol 99:2725-2730.

Silvanto J, Muggleton N, Lavie N, Walsh V (2009) The perceptual and functional consequences of parietal top-down modulation on the visual cortex. Cereb Cortex 19:327-330.

Smith AT, Singh KD, Greenlee MW (2000) Attentional suppression of activity in the human visual cortex. Neuroreport 11:271-277.

Smith SM (2002) Fast robust automated brain extraction. Hum Brain Mapp 17:143-155.

Sylvester CM, Jack AI, Corbetta M, Shulman GL (2008) Anticipatory suppression of nonattended locations in visual cortex marks target location and predicts perception. J Neurosci 28:6549-6556.

Walsh V, Cowey A (2000) Transcranial magnetic stimulation and cognitive neuroscience. Nat Rev Neurosci 1:73-79.

Wassermann EM (1998) Risk and safety of repetitive transcranial magnetic stimulation: report and suggested guidelines from the International Workshop on the Safety of Repetitive Transcranial Magnetic Stimulation, June 5-7, 1996. Electroencephalogr Clin Neurophysiol 108:1-16.

Woolrich MW, Ripley BD, Brady M, Smith SM (2001) Temporal autocorrelation in univariate linear modelling of FMRI data. Neuroimage 14:1370-1386.

Woolrich MW, Behrens TE, Beckmann CF, Jenkinson M, Smith SM (2004) Multi-level linear modeling for FMRI group analysis using Bayesian inference. Neuroimage 21:1732-1747.

Worsley KJ, Evans AC, Marrett S, Neelin P (1992) A three-dimensional statistical analysis for $\mathrm{CBF}$ activation studies in human brain. J Cereb Blood Flow Metab 12:900-918. 